\title{
New results on stabilization of networked control systems with packet disordering ${ }^{\text {th }}$
}

\author{
Andong Liu, ${ }^{\mathrm{a}, *}$, Wen-an Zhang ${ }^{\mathrm{a}}$, Li Yua ${ }^{\mathrm{a}}$, Steven Liu ${ }^{\mathrm{b}}$, Michael Z. Q. Chen ${ }^{\mathrm{c}}$ \\ ${ }^{a}$ Department of Automation, Zhejiang University of Technology, Zhejiang Provincial United Key Laboratory of Embedded Systems, Hangzhou 310023, P.R. China \\ ${ }^{b}$ Department of Electrical and Computer Engineering, University of Kaiserslautern, Kaiserslautern 67663, Germany \\ ${ }^{c}$ Department of Mechanical Engineering, The University of Hong Kong, Hong Kong
}

\begin{abstract}
In this paper, the stabilization problem is studied for a class of networked control systems (NCSs) with delays, packet disordering and packet dropouts. A new packet reordering method is presented to deal with packet disordering and choose the newest control input. A relationship between the reordered packet over two consecutive sampling intervals is given for the NCS with both time delays and packet dropouts. A sufficient condition for the NCS to be exponentially stable is presented by using the average dwelltime method. Finally, an illustrative example is given to demonstrate the effectiveness of the proposed method.
\end{abstract}

Keywords: Networked control systems, Network-induced delays, Packet disordering, Packet dropouts

\section{Introduction}

Network-induced delay and packet dropout are two main problems in networked control system (NCS), and have attracted much research interest, see for example [1], [2], [3], [4], [5] and the references therein. Compared with constant delays, the time-varying one is more difficult to treat, especially, when the delay is larger than one sampling period (long delay). Since the delay may be larger than one sampling period, more than one control signals may arrive at the actuator during one sampling interval. Moreover, the transmission of data packets may not necessarily follow a "first send first arrive" principle [6]. This means that the newest control signal may arrive at the actuator before the older one, this is the so-called packet disordering problem [7], [8]. The modeling and stability analysis of NCSs with packet disordering has attracted much research attention, see for example [9], [10], [11], [12] and [13]. Due to limited network transmission capacity, packet dropouts are usually inevitable. Some results on NCSs concerning both delay and packet dropout issues were presented in [14], [15], [16], [17] and [18]. It should be pointed out that the packet disordering problem is not considered in the aforementioned results for NCSs in the presence of delays and packet dropouts. In [19], $H_{\infty}$ filtering problem has been investigated for NCSs with delays, packet disordering and packet dropouts. However, the explicit expression for how to choose the newest data signal was not given in [19].

On the other hand, due to the time-varying delays and packet dropouts, the number of available control signals at the ac-

\footnotetext{
This work was supported by the National Natural Science Foundation of China under Grants 61273117, 61374053, and 61403344.

${ }^{*}$ Corresponding author. Tel.: +86 571 85290531; fax: +86 57185290567

Email addresses: lad-andrew@hotmail . com (Andong Liu ), wazhg@hotmail.com (Wen-an Zhang), lyu@zjut.edu.cn (Li Yu), sliu@eit.uni-kl.de (Steven Liu), and mzqchen@gmail.com (Michael Z. Q. Chen)
}

tuator vary over different sampling intervals. Therefore, the NCS is naturally a switched system with a group of subsystems describing various system dynamics on different sampling intervals ([20], [21], [22]). In response to the above discussion, we investigate the stability analysis problem for a class of NCSs with delays and packet dropouts, and focus on solving the packet disordering problem and the switched dynamic caused by long delay and packet dropout. The main contributions of the paper are as follows: 1) A new packet reordering method is proposed to deal with packet disordering and choose the newest control signal. 2) A relationship between the reordered packet over two consecutive sampling intervals is given for the NCS with both network-induced delays and packet dropouts. 3) A sufficient condition for exponential stability of the NCS is derived by using the average dwell-time method. Finally, an example is given to demonstrate the effectiveness of the proposed method.

\section{Modeling of the NCS}

The plant in the NCS is described by the following continuous-time linear system model

$$
\dot{x}(t)=A_{p} x(t)+B_{p} u(t)
$$

where $x(t) \in R^{n}$ is the system state, $u(t) \in R^{m}$ is the control input, $A_{p}$ and $B_{p}$ are constant matrices with appropriate dimensions. The sensor is time-driven with sampling period $T$, the controller is event-driven, the actuator is time-driven and has a receiving buffer containing the most recent data packet from the controller, time delay $\tau_{k}$ is assumed to be bounded by $0 \leq \tau_{k} \leq d T$, where $d$ is a known finite integer. Since the actuator is time-driven, one has $\tau_{k} \in \mathcal{N}_{1}=\{0,1,2, \cdots, d\} T$.

For simplicity, we first consider only the delay issue. Since the network-induced delay may be larger than one sampling 
period, more than one control signals may arrive at the actuator during one sampling interval, but only one control signal is adopted by the actuator. Then, the problem is how to choose the newest control signal. Moreover, packet disordering problem arises when the newest packet arrive at the actuator before the older ones. A time diagram of the signal transmission is illustrated in Figure 1, in which it is assumed that $d=3$, and at most four control signals may arrive at the actuator during one sampling interval. Furthermore, control signal $u(k+2)$ arrives at the actuator earlier than $u(k+1)$. Thus, $u(k+2)$ is adopted at time $(k+3) T$. This phenomenon is called packet disordering. In order to use the newest control signal, $u(k+1)$ will be discarded.
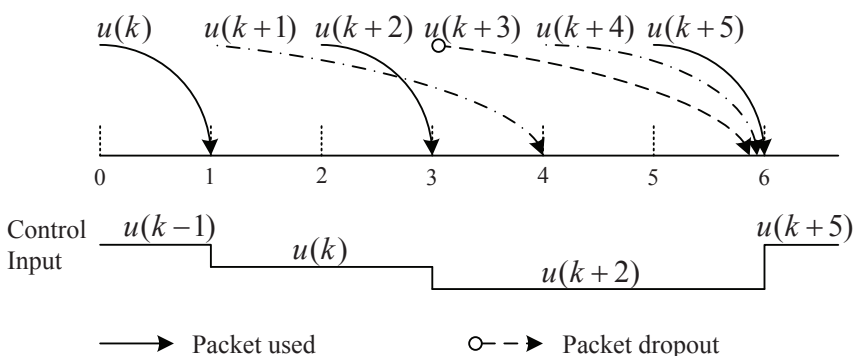

Fig.1. Time diagram of signal transmitting in the NCS.

By the aforementioned analysis, it can be seen that the adopted control signal may take values in $\{u(k-d+1), \cdots$, $u(k-1), u(k)\}$ at sampling instant $k T$, which will result in $d+1$ different dynamics of the system. Moreover, during the sampling interval $[k T,(k+1) T)$, the system dynamics are actually determined by $\left\{\tau_{k-d+1}, \cdots, \tau_{k-1}, \tau_{k}\right\}$. Therefore, we use a vector $\tau(k)=\left[\tau_{k-d+1}, \cdots, \tau_{k-1}, \tau_{k}\right]$ to represent the control signal that is applied at the actuator, and define a vectorvalued function $f: \tau(k) \rightarrow \sigma(k)$ to map $\tau(k)$ into a scalar $\sigma(k) \in \mathcal{N}_{2}=\{0,1, \cdots, d\}$. The expression of $\sigma(k)$ is given in detail as follows

$$
\sigma(k)=\left\{\begin{array}{cc}
0, & \tau_{k}=0, \tau_{k-j} \in \mathcal{N}_{1}, j=1,2, \cdots, d-1 \\
1, & \tau_{k} \geq T, \tau_{k-1} \leq T, \tau_{k-j} \in \mathcal{N}_{1} \\
& j=2, \cdots, d-1 \\
2, & \tau_{k-i} \geq(i+1) T, i=0,1, \tau_{k-2} \leq 2 T \\
& \tau_{k-j} \in \mathcal{N}_{1}, j=3, \cdots, d-1 \\
\vdots & \vdots \\
d, & \tau_{k-i} \geq(i+1) T, i=0,1, \cdots d-1
\end{array}\right.
$$

and then the adopted control signal at time $k T$ is $u(k-\sigma(k))$. Equation (2) presents an explicit logic expression for how to choose the newest control signal and eliminating the impact of packet disordering. Also, $\sigma(k)$ can be given concisely as follows

$$
\sigma(k)=\min \left\{i \mid \tau_{k-i}-i T \leq 0, i=0,1, \cdots, d\right\}
$$

Then, we have the following proposition.

Proposition 1. If $\sigma(k)=r$, then $\sigma(k+1) \leq r+1$.
Proof. Let $\sigma(k+1)=\min \left\{j \mid \tau_{k+1-j}-j T \leq 0, j=0,1, \cdots, d\right\}$. If $\sigma(k)=r$, it follows from (3) that $\tau_{k-r}-r T \leq 0$ and $\tau_{k-i}-i T>$ 0 for $i<r$. From the above analysis, it can be obtained that $\tau_{k+1-(r+1)}-(r+1) T<0$, which infers that $\sigma(k+1) \leq r+1$. This completes the proof.

Remark 1. The packet disordering can be effectively eliminated by applying the mechanism given in (3). Assuming that the packets of time $(k-i-1) T$ and $(k-i) T$ are disordering, then one obtains $\tau_{k-i-1} \geq \tau_{k-i}+2 T$. Therefore, if $\tau_{k-i-1}-(i+1) T \leq 0$, it must be $\tau_{k-i}-i T<0$, which means that the actuator will use the newest packet of time $(k-i) T$ and discard packet of time $(k-i-1) T$.

Let $\theta_{k}$ be the number of consecutive packet dropout with $\theta_{k} \in[0, s], s \leq d$ at time $k T$, and $\tau_{k}=d T$ if the packet of time $k T$ is lost. A time diagram of the signal transmitting with both time delay and packet dropout is illustrated in Figure 1. In view of (2), it is difficult to handle packet dropout in a unified framework because $\sigma(k) \in\{0,1, \cdots, d+s\}$. Inspired by Equation (3), the following result can be derived with both time delays and packet dropouts

$$
\sigma(k)=r+\theta_{k-r}
$$

where $r=\min \left\{i \mid \tau_{k-i}-\left(i+\theta_{k-i}\right) T \leq 0, i=0,1, \cdots, d\right\}$.

Remark 2. If there is no packet dropout during sampling interval $[(k-d) T, k T]$, one has $\theta_{k-i}=0$ for $i \in\{0,1, \cdots, d\}$. Then (4) equals to (3). If $\theta_{k-r} \neq 0$, the packets of time $(k-r) T$ to $\left(k-r-\theta_{k-r}\right) T$ are dropout and $\tau_{k-i}-\left(i+\theta_{k-i}\right) T>0$ for $0 \leq i<r$, which means that the packet of time $(k-i) T$ for $0 \leq i<r$ is not successfully delivered during interval $((k-r) T, k T]$. Therefore, Equation (4) shows that the actuator always uses the newest packet during interval $[(k-d-s) T, k T]$. Similar to Proposition 1, the following proposition can be obtained.

Proposition 2. If $\sigma(k)=r+\theta_{k-r}, 0 \leq \theta_{k-r} \leq s$, then $\sigma(k+1) \leq$ $r+\theta_{k-r}+1$ and $\sigma(k+1) \neq r+2, \cdots, r+\theta_{k-r}$.

Proof. By using a similar method to the proof of Proposition 1, it can be easily obtained that $\sigma(k+1) \leq r+\theta_{k-r}+1$. Next, we will prove $\sigma(k+1) \neq r+2, \cdots, r+\theta_{k-r}$.

If the packet of time $(k-r) T$ is successfully delivered during interval $[(k-d) T, k T]$, it means $\theta_{k-r}=0$. Thus, $\sigma(k+1) \leq r+1$. If the packet of time $(k-r) T$ is dropout, then one has

$$
r+\theta_{k-r}=r+1+\theta_{k-r-1}=\cdots=r+\theta_{k-r}+\theta_{k-r-\theta_{k-r}}
$$

which implies that $\theta_{k-r-\theta_{k-r}}=0$. If the packet at time $(k-r+1) T$ is dropout, the following result can be obtained by (5)

$$
r-1+\theta_{k-r+1}=r+\theta_{k-r}
$$

It follows from (6) that $\sigma(k)=r-1+\theta_{k-r+1}$. However, it is inconsistent with condition $\sigma(k)=r+\theta_{k-r}$. Therefore, if the packet of time $(k-r) T$ is dropout, the packet at time $(k-r+1) T$ must be successfully delivered.

Let $\sigma(k+1)=\bar{j}+\theta_{k+1-\bar{j}}$. If $\tau_{k+1-j}-\left(j+\theta_{k+1-j}\right) T>0$ for $0 \leq j \leq r$, then $\bar{j}=r+1$ and $\sigma(k+1)=r+\theta_{k-r}+1$. If there 
exists a $j$ for $0 \leq j \leq r$ satisfied $\tau_{k+1-j}-\left(j+\theta_{k+1-j}\right) T \leq 0$, it follows from (5) and (6) that $\bar{j}+\theta_{k+1-\bar{j}} \leq r$ because the packet at time $(k-r+1) T$ must be successfully delivered. Therefore, $\sigma(k+1) \leq r$ and $\sigma(k+1) \neq r+1, r+2, \cdots, r+\theta_{k-r}$. The proof is thus completed.

Remark 3. If there is no delay in the communication network, then $\tau_{k}=0$. Thus, Equation (4) becomes $\sigma(k)=\theta_{k}$. It can be known that if the packet of time $k T$ is successfully delivered, then $\sigma(k)=0$, otherwise $\sigma(k)=\gamma_{k}$. Therefore, if $\sigma(k)=\theta_{k}$, $0 \leq \theta_{k} \leq m$, then $\sigma(k+1)=0$ or $\sigma(k+1)=\theta_{k}+1$.

Remark 4. In [19], an $H_{\infty}$ filtering problem has been investigated for NCSs both with random delays and packet dropouts. A logical data packet processor is introduced to choose the newest data signal from the network to the filter. However, the explicit expression for how to choose the newest data signal was not given in [19]. In this paper, the explicit expression for how to choose the newest data signal is given in (4), which leads to a new modeling approach, and some new results are also given in Proposition 2.

Let $\mathcal{N}_{3}=\{0,1, \cdots, d+s\}$, it can be known from (4) that $\sigma(k) \in \mathcal{N}_{3}$. Denote $A=e^{A_{p} T}$ and $B=\int_{0}^{T} e^{A_{P} t} d t B_{p}$, then the NCS under consideration is modeled as the following discretetime delay system

$$
x(k+1)=A x(k)+B u(k-\sigma(k))
$$

Let $\Lambda_{i \sigma(k)}=\left\{\begin{array}{ll}I, & i=\sigma(k) \\ 0, & i \neq \sigma(k)\end{array}, i \in \mathcal{N}_{3}\right.$, then system (7) can be represented as follows

$$
x(k+1)=A x(k)+\sum_{i=0}^{d+s} \bar{B}_{i \sigma(k)} u(k-i)
$$

where $\bar{B}_{i \sigma(k)}=\Lambda_{i \sigma(k)} B$. Let $X(k)=\left[\begin{array}{lll}x^{T}(k) & u^{T}(k-1) \quad \cdots\end{array}\right.$ $\left.u^{T}(k-d-s+1) \quad u^{T}(k-d-s)\right]^{T}, \quad$ system (8) can be rewritten as the following switched system model

$$
X(k+1)=G_{\sigma(k)} X(k)+H_{\sigma(k)} u(k)
$$

where

$$
\begin{aligned}
G_{\sigma(k)} & =\left[\begin{array}{ccccc}
A & \bar{B}_{1 \sigma(k)} & \cdots & \bar{B}_{(d+s-1) \sigma(k)} & \bar{B}_{(d+s) \sigma(k)} \\
0 & 0 & \cdots & 0 & 0 \\
0 & I & \cdots & 0 & 0 \\
\vdots & \vdots & \ddots & \vdots & \vdots \\
0 & 0 & \cdots & I & 0
\end{array}\right], \\
H_{\sigma(k)} & =\left[\begin{array}{ccccc}
\bar{B}_{0 \sigma(k)}^{T} & I & 0 & \cdots & 0
\end{array}\right]^{T} .
\end{aligned}
$$

Then, the objective is to design a stabilizing controller $u(k)=$ $K X(k)$ for the NCS (9) via switched system approach. Thus, the closed-loop system of (9) is given as follows

$$
X(k+1)=\Phi_{\sigma(k)} X(k)
$$

where $\Phi_{\sigma(k)}=G_{\sigma(k)}+H_{\sigma(k)} K$.
Remark 5. The subsystem of (10) activated during the sampling interval $[k T,(k+1) T)$ is determined by $\tau(k)$. When $\tau(k)$ takes a certain value, the system (10) resides in the corresponding subsystem. Therefore, system (10) is essentially a switched system with a finite number of subsystems. By using the vectorvalued function $f: \tau(k) \rightarrow \sigma(k), \sigma(k)$ is used as the switching signal of the closed-loop NCS (10).

\section{Stability analysis and controller design}

Before proceeding further, some useful definitions are introduced.

Definition 1. The NCS (10) is said to be exponentially stable, if there exist positive constants $c$ and $\alpha<1$ such that the solution of system (10) satisfies

$$
\|X(k)\|=c \alpha^{k}\left\|X_{0}\right\|
$$

for any initial state $X_{0}=X(0) \in R^{(d+1) n}$, where $X(0)=$ $\left[\begin{array}{ccccc}x^{T}(0) & u^{T}(-1) & \cdots & u^{T}(-d-s+1) & u^{T}(-d-s)\end{array}\right]^{T}$, $u(i)=0, i=-1, \cdots,-d-s+1,-d-s$.

Definition 2 [12] . For any switching signal $\sigma(k)$ and any $k \geq$ 1 , let $N_{\sigma}[0, k)$ be the number of switching of $\sigma(k)$ over the interval $[0, k)$. If $N_{\sigma}[0, k) \leq N_{0}+k / T_{\alpha}$ holds for $N_{0} \geq 0$ and $T_{\alpha} \geq 0$, then $T_{\alpha}$ is called the average dwell time and $N_{0}$ is the chatter bound.

For simplicity, but without the loss of generality, we choose $N_{0}=0$ in the following development. A sufficient condition for the exponential stability of the closed-loop NCS (10) is now presented in the following theorem.

Theorem 1. For given positive scalars $\lambda<1$ and $\mu>1$, if there exist matrices $P_{i}>0, K, i=0,1, \cdots d+m$ of appropriate dimensions such that the following inequalities

$$
\begin{aligned}
& \Phi_{i}^{T} P_{i} \Phi_{i}-\lambda^{2} P_{i} \leq 0 \\
& P_{a} \leq \mu P_{b}, \quad \forall a, b \in \mathcal{N}_{3} \\
& T_{\alpha}>T_{\alpha}^{*}=\frac{\operatorname{In} \mu}{2 \operatorname{In} \lambda^{-1}}
\end{aligned}
$$

hold, then the closed-loop NCS (10) is exponentially stable and ensures a decay rate $\alpha=\lambda \mu^{\frac{1}{2 T_{\alpha}}}$.

Proof. The proof can be followed by applying some similar procedures to those in [12], and is thus omitted.

Theorem 2. For given positive scalars $\lambda<1$ and $\mu>1$, if there exist matrices $Z, V, Q_{i}>0, i=0,1, \cdots, d+s$ of appropriate dimensions such that (13) is true and that the following LMIS

$$
\begin{aligned}
& {\left[\begin{array}{cc}
-\lambda^{2} Q_{i} & * \\
G_{i} Z+H_{i} V & -Z-Z^{T}+Q_{i}
\end{array}\right]<0} \\
& Q_{a} \leq \mu Q_{b}, \quad \forall a, b \in \mathcal{N}_{3}
\end{aligned}
$$

hold, then the closed-loop NCS (10) is exponentially stable and ensure a decay rate $\alpha=\lambda \mu^{\frac{1}{2 T_{\alpha}}}$ with controller gain $K=V Z^{-1}$. 
Proof. By Lemma 1 in [12], inequality (11) is true if there exists a matrix $Y$ such that the following matrix inequality

$$
\left[\begin{array}{cc}
-\lambda^{2} P_{i} & * \\
Y^{T} G_{i}+Y^{T} H_{i} K & -Y-Y^{T}+P_{i}
\end{array}\right]<0
$$

holds. Inequality (16) implies that $Y$ is invertible. Denote $Z=Y^{-1}, V=K Z, Q_{i}=Z^{T} P_{i} Z$. Then, the LMI (14) can be obtained by pre- and post-multiplying the inequality (16) by $\operatorname{diag}\left\{Z^{T}, Z^{T}\right\}$ and $\operatorname{diag}\{Z, Z\}$, respectively. Pre- and postmultiplying the inequality (12) by $Z^{T}$ and $Z$, respectively, we obtain inequality (15). The proof is thus completed.

Remark 6. The parameters $\lambda$ and $\mu$ should be chosen as small as possible to yield a smaller $\alpha$ and $T_{\alpha}^{*}$, which results in better performance and makes the condition (13) easier to be satisfied.

\section{Numerical example}

In this section, a simulation example is given to demonstrate the effectiveness of the developed method.

Consider an inverted pendulum system with delayed control input [2], its state-space model is given by

$$
\dot{x}(t)=\left[\begin{array}{cc}
0 & 1 \\
\frac{3(M+m) g}{l(4 M+m)} & 0
\end{array}\right] x(t)+\left[\begin{array}{c}
0 \\
-\frac{3}{l(4 M+m)}
\end{array}\right] u(t)
$$

where $x=\left[\begin{array}{cc}\theta & \dot{\theta}\end{array}\right]^{T} ; \theta$ is the angle of the pendulum from the top vertical; $M$ and $m$ are the masses of the cart and the pendulum, respectively; $l$ is the half length of the pendulum; $g$ is the acceleration due to gravity. The parameters are selected as $M=1.096 \mathrm{~kg}, \mathrm{~m}=0.109 \mathrm{~kg}, l=0.25 \mathrm{~m}$, and $g=9.8 \mathrm{~m} / \mathrm{s}^{2}$. By assuming the sampling time to be $T=0.06 \mathrm{~s}$, the discretized model for the pendulum system is given by

$$
x(k+1)=\left[\begin{array}{ll}
1.0573 & 0.0611 \\
1.9284 & 1.0573
\end{array}\right] x(k)+\left[\begin{array}{l}
-0.0049 \\
-0.1633
\end{array}\right] u(k)
$$

First, we consider only the packet disordering issue. The time delay $\tau_{k}$ is bounded by $\tau_{k} \leq 2 T$. Then, one obtains $d=2$, which infers that at most three control signals can be involved during one sampling period. It can be seen from Equation (3) that totally three subsystems are involved and $\sigma(k) \in\{0,1,2\}$. Figure 2 depicts the distribution of delay $\tau_{k}$, where the asterisk one denotes time delay, the circle one denotes packet disordering. By the discussion of Remark 1, if $\tau_{k-1}-\tau_{k}>T$, it will cause packet disordering. It follows from the given delay sequence $\tau_{k}$ that packet disordering is inevitable in this case and occurs 9 times. By using Equation (3), the subsystems are activated by the sequence $\sigma(k)$ which is shown in Figure 2. It can be seen from Figure 2 that the number of switching for sequence $\tau_{k}$ and $\sigma(k)$ are 37 and 40, respectively. Moreover, the times of switching are also different. It shows that packet disordering has a significant effect on the system dynamics.

Choosing the initial condition $x(0)=\left[\begin{array}{ll}0.1 & 0\end{array}\right]^{T}, \mu=$ 1.05, $\lambda=0.98$. By the switching sequence $\sigma(k)$ of the subsystems and the definition of the average dwell time, we have $T_{\alpha}=100 / 40=2.5$, and therefore the condition $T_{\alpha}>T_{\alpha}^{*}=\operatorname{In} \mu / 2 \operatorname{In}(1 / \lambda)=1.2075$ is also satisfied. By Theorem 2, one obtains the controller gain $K=$ $\left[\begin{array}{llll}22.5211 & 4.0207 & -0.2931 & -0.2378\end{array}\right]$. Thus, the NCS controlled by the designed controller via network is exponentially stable and has a decay rate $\alpha=0.992$. The simulation results are shown in Figure 3, where the dotted line and solid line describe the state trajectories of the NCS without packet reordering and with packet reordering, respectively. It can be seen from Figure 3 that the packet disordering has a negative effect on the performance of the NCS.
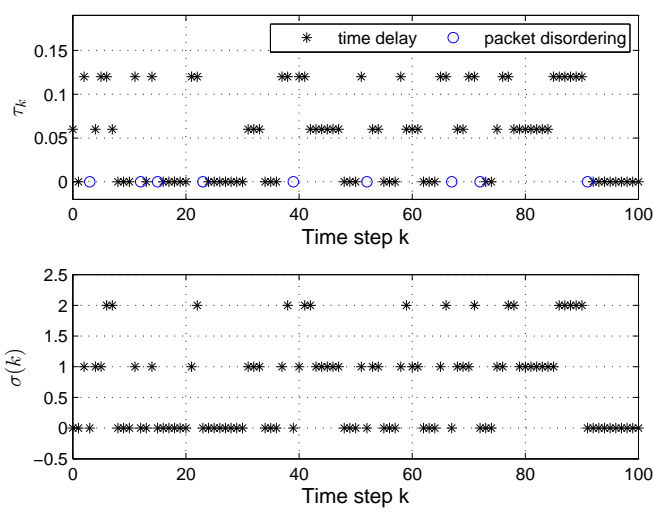

Fig.2. Distribution of $\tau_{k}$ and $\sigma(k)$.
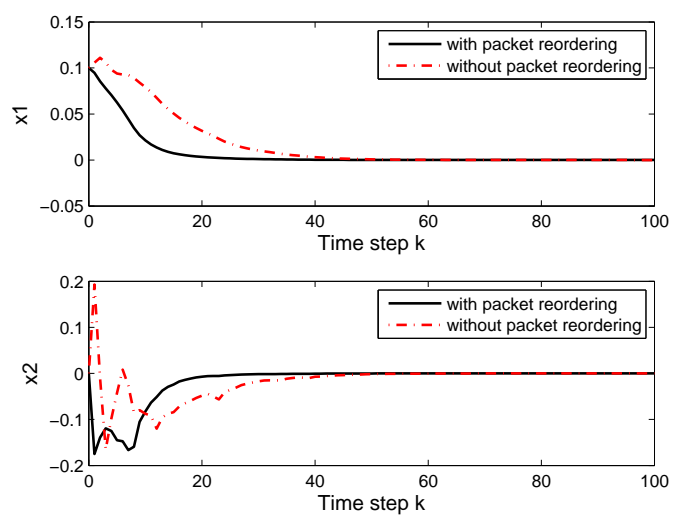

Fig.3. State trajectories of the NCS.

In what follows, it is assumed that the maximum consecutive packet dropout is 1 . Then, one obtains $d=2$ and $s=1$. It can be seen from Equation (4) that totally four subsystems are involved and $\sigma(k) \in\{0,1,2,3\}$. Figure 4 depicts the distribution of the delays and packet dropouts, where the asterisk one denotes time delay, the circle one denotes packet disordering and the right triangle denotes packet dropout. It can be seen from Figure 4 that 12 packets are dropout and packet disordering occurs 15 times. By using Equation (4), the subsystems are activated by the sequence $\sigma(k)$ which shows in Figure 4. It can be seen from Figure 4 that the number of switching for sequence $\tau_{k}$ and $\sigma(k)$ are 54 and 65, respectively. Moreover, the times of switching are also different. These phenomena are caused by packet disordering and packet dropout. 
By choosing the same parameters $x(0), \mu$ and $\lambda$, one obtains $T_{\alpha}=100 / 65=1.5385$. Therefore, the condition $T_{\alpha}>T_{\alpha}^{*}$ is also satisfied. By Theorem 2, one obtains the controller gain $K=\left[\begin{array}{lllll}25.6369 & 4.5785 & -0.3927 & -0.3254 & -0.2552\end{array}\right]$. Thus, the NCS controlled by the designed controller via network is exponentially stable and has a decay rate $\alpha=0.992$. The simulation results are shown in Figure 5, where the dotted line and solid line describe the state trajectories of the NCS without packet reordering and with packet reordering, respectively. It can be seen from Figure 5 that the packet disordering has a detrimental effect on the performance of the NCS.
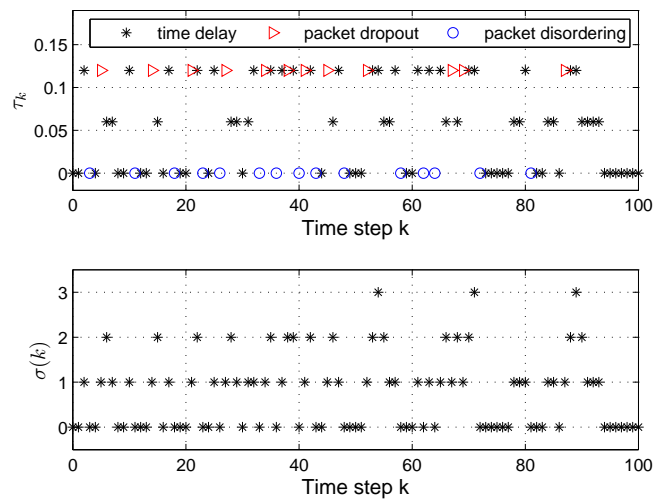

Fig.4. Distribution of $\tau_{k}, \sigma(k)$ and packet dropout.
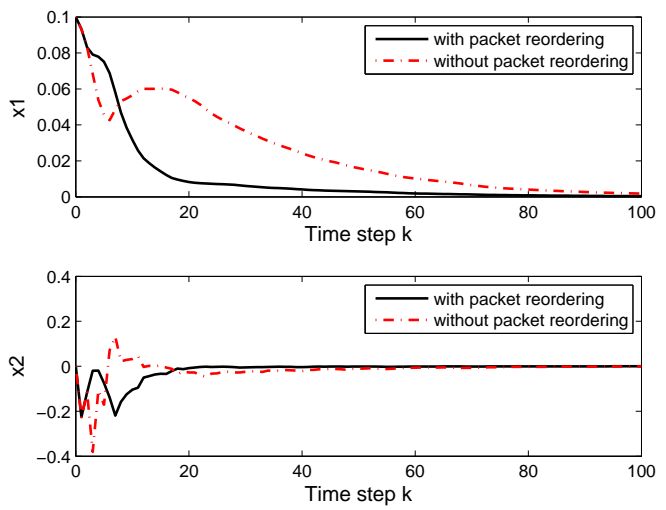

Fig.5. State trajectories of the NCS.

\section{Conclusion}

In this paper, a switched system approach was presented for a class of NCSs with time delays, packet disordering and packet dropouts. A new approach was proposed to deal with the detrimental effect of packet disordering, and the NCS was modeled as a switched system, which can fully describe the phenomenon of packet disordering. Two propositions are proposed for the relationship of the reordered packet. The condition for exponential stability and controller design procedure were presented for the closed-loop NCS. The effectiveness of the proposed method was illustrated by a numerical example.

\section{References}

[1] Zhang L., Gao H., \& Kaynak O. Network-induced constraints in networked control systems-A survey. IEEE Transactions on Industrial Informatics, 2013, 9(1): 403-416.

[2] Gao, H., \& Chen, T. New results on stability of discrete-time systems with time-varying state delay. IEEE Transactions on Automatic Control, 2007, 52(2): 328-334.

[3] Garcia, E., \& Antsaklis, P.J. Model-based event-triggered control for systems with quantization and time-varying network delays. IEEE Transactions on Automatic Control, 2013, 58(2): 422-434.

[4] Xiong J., \& Lam J. Stabilization of linear systems over networks with bounded packet loss. Automatica, 2007, 43(1): 80-87.

[5] Yang F., \& Han Q.L. $H_{\infty}$ control for networked systems with multiple packet dropouts. Information Sciences, 2013, 252: 106-117.

[6] Zhao, Y.B., Kim, J., Liu, G.P., \& Rees, D. Compensation and stochastic modeling of discrete-time networked control systems with data packet disorder. International Journal of Control Automation and Systems, 2012, 10(5): 1055-1063.

[7] Liu, A., Yu, L., \& Zhang, W.A. One-step receding horizon $H_{\infty}$ control for networked control systems with random delay and packet disordering. ISA Transactions, 2011, 50(1): 44-52.

[8] Liu, A., Yu, L., \& Zhang, W.A. $H_{\infty}$ control for network-based systems with time-varying delay and packet disordering. Journal of the Franklin institute, 2011, 348(5): 917-932.

[9] Cloosterman, M.B.G., van de Wouw, N., Heemels, W.P.M.H., \& Nijmeijer, H. Stability of networked control systems with uncertain time-varying delays. IEEE Transactions on Automatic Control, 2009, 54(7): 15751580 .

[10] Cloosterman, M.B.G., Hetel, L., van de Wouw, N., Heemels, W.P.M.H., Daafouz, J., \& Nijmeijer, H. Controller synthesis for networked control systems. Automatica, 2010, 46(10): 1584-1594.

[11] Li, J., Zhang, Q., Yu, H., \& Cai, M. Real-time guaranteed cost control of MIMO networked control systems with packet disordering. Journal of Process Control, 2011, 21(6): 967-975.

[12] Zhang, W.A., \& Yu, L. New approach to stabilisation of networked control systems with time-varying delays. IET Control Theory and Applications, 2008, 2(12): 1094-1104.

[13] Zhang, W.A., \& Yu, L. A robust control approach to stabilization of networked control systems with time-varying delays. Automatica, 2009, 45(10): 2440-2445.

[14] García-Rivera M., \& Barreiro A. Analysis of networked control systems with drops and variable delays. Automatica, 2007, 43(12): 2054-2059.

[15] Wang, Z., Shen, B., Shu, H., \& Wei, G. Quantized $H_{\infty}$ control for nonlinear stochastic time-delay systems with missing measurements. IEEE Transactions on Automatic Control, 2012, 57(6): 1431-1444.

[16] Yang R., Shi P., Liu G.P., \& Gao H. Network-based feedback control for systems with mixed delays based on quantization and dropout compensation. Automatica, 2011, 47(12): 2805-2809.

[17] Xia Y., Liu G.P., Fu M., \& Rees D. Predictive control of networked systems with random delay and data dropout. IET Control Theory and Applications, 2009, 3(11): 1476-1486.

[18] Yang R., Liu G.P., Shi P., Thomas C., \& Basin M.V. Predictive output feedback control for networked control systems. IEEE Transactions on Industrial Electronics, 2014, 61(1): 512-520.

[19] Zhang X.M., \& Han Q.L. Network-based $H_{\infty}$ filtering for discrete-time systems. IEEE Transactions on Signal Processing, 2012, 60(2): 956-961.

[20] Hetel, L., Daafouz, J., \& Lung, C. Analysis and control of LTI and switched systems in the digital loops via an event-based modeling. International Journal of Control, 2008, 81(7): 1125-1138.

[21] Lin, H., \& Antsaklis, P.J. Stability and persistent disturbance attenuation properties for a class of networked control systems: Switched system approach. International Journal of Control, 2005, 78(18): 1447-1458.

[22] Wang, R., Liu, G.P., Wang, W., Rees, D., \& Zhao, Y.B. $H_{\infty}$ control for networked predictive control systems based on the switched Lyapunov function method. IEEE Transactions on Industrial Electronics, 2010, 57(10): 3565-3571. 